\title{
INTRINSIC STATES OF DEFORMED NUCLEI IN THE HARTREE-FOCK (HF) APPROXIMATION
}

\author{
R. MUTHUKRISHNAN \\ Department of Physics, University of Michigan, Ann Arbor, Michigan, U.S.A. ${ }^{\dagger}$
}

Received 7 September 1966

\begin{abstract}
This paper deals with an application of the HF method to deformed nuclei. The two nucleon interaction used is a non-local separable potential which acts only in relative s-states. The HF equations are solved in the matrix formulation. Calculations are performed for ${ }^{16} \mathrm{O},{ }^{20} \mathrm{Ne}$, ${ }^{24} \mathrm{Mg},{ }^{28} \mathrm{Si},{ }^{32} \mathrm{~S},{ }^{36} \mathrm{Ar}$ and ${ }^{40} \mathrm{Ca}$. The $4 \mathrm{p}-4 \mathrm{~h}$ state of ${ }^{16} \mathrm{O}$ is also investigated. Solutions corresponding to different kinds of deformation are investigated. Single-particle energies and wave functions are obtained. The intrinsic quadrupole moments are also calculated.
\end{abstract}

\section{Introduction}

The shell-model description of nuclei has been very successful in correlating a large amount of experimental data. Even some of the collective properties of deformed nuclei can be interpreted in terms of a model ${ }^{1}$ ) which is based on the concepts of shell model, viz. "unified model". The theoretical basis for the shell model is the Hartree-Fock ${ }^{2}$ ) picture where one obtains a self-consistent one-body potential in which the nucleons are moving independently of each other. The derivation of this potential from two-nucleon interaction in the HF approximation is very difficult due to the presence of the hard core in the nucleon-nucleon force. In recent years it has been shown ${ }^{3}$ ) that the two-nucleon scattering data can be fitted with velocitydependent potentials, which have the saturation property needed to explain the behaviour of binding energy of nuclei as a function of mass number. Moreover these potentials are suitable for HF calculations. Such calculations have been reported ${ }^{4}$ ) for closed shell nuclei. HF calculations ${ }^{5}$ ), where only part of the nucleons in the nuclei are included, have been carried out for $2 \mathrm{~s} 1 \mathrm{~d}$ shell nuclei using phenomenological interactions. Several nuclei in $2 \mathrm{~s} 1 \mathrm{~d}$ shell have rotational level structure, and in many cases experimental information about deformation and intrinsic quadrupole moment are available. Unlike in the case of closed shell nuclei, the HF approximation for non-closed shell nuclei enables one to study the intrinsic properties.

The rotational collective aspect of nuclear excitations is well explained by "unified model" ${ }^{1}$ ). The model assumes an average potential which is non-spherical, in which the single-particle levels are filled according to the Pauli principle. The collective motion is obtained through the rotation of the non-spherical wcll. Hill and Wheeler ${ }^{6}$ )

$\dagger$ Research supported by the National Science Foundation. 
and Peirls and Yaccoz ${ }^{7}$ ) have given the mathematical description of the unified model. The intrinsic wave function is assumed to be a Slater determinant obtained by filling the particles in the non-spherical well. Due to the fact that the well is deformed an angle $\Omega$ is assigned to specify the orientation of the axis. But $\Omega$ is arbitrary and hence there is a set of degenerate wave functions which differ only in the value of $\Omega$. So one can construct a more general wave function,

$$
\Psi=\int f(\Omega) \Phi(1, \ldots N ; \Omega) \mathrm{d} \Omega .
$$

Peirls and Yaccoz show (for the case of axial symmetry) that by minimizing the expectation value of the Hamiltonian between the state $\Psi$, with respect to $f(\Omega)$ the value of the energy can be lowered, and for a spherically symmetric Hamiltonian,

$$
f(\Omega) \propto Y_{L}^{M}(\theta, \varphi),
$$

where $Y_{L}^{M}(\theta, \varphi)$ is a spherical harmonics, and angles $\theta, \varphi$ specify the symmetry axis with respect to a space fixed axis. With this value for $f(\Omega)$ the wave function $\Psi$ has the total angular momentum as a good quantum number. This is also the way in which wave functions are constructed in Elliott's $\mathrm{SU}_{3}$ model $^{8}$ ).

There have been many phenomenological average deformed potentials discussed in the literature ${ }^{9}$ ), and the most successful one among them is that of Nilsson. The Nilsson model assumes an axially symmetric deformation. Although this model is used extensively in calculation of nuclear properties, the empherical nature of the model makes it difficult to predict the nature of the deformation viz, prolate, oblate or asymmetric. Thus an HF calculation will complement the Nilsson model, and a comparison of ordering of levels and wave functions can be made.

The two-nucleon interaction used is a non-local separable force which acts only in relative s-states. The parameters of the force (see sect. 3 ) are chosen to fit the binding energy and density of nuclear matter. HF calculations for closed shell nuclei using this force have been reported elsewhere ${ }^{10}$ ), and the results were qualitatively in good agreement with experiment. Due to the fact that this interaction is purely central, the spin-orbit splitting of single-particle levels were not obtained. Although the tensor interaction plays an important role, calculations such as the present one with a much simplified force can enable one to learn about the validity of the $\mathrm{HF}$ method. This might lead the way for future calculations with more sophisticated interactions.

The calculations reported here are performed for nuclei in the $2 \mathrm{~s} 1 \mathrm{~d}$ shell $\left({ }^{20} \mathrm{Ne}\right.$, ${ }^{24} \mathrm{Mg},{ }^{28} \mathrm{Si},{ }^{32} \mathrm{~S}$ and ${ }^{36} \mathrm{Ar}$ ) with mass number $A=4 n$ ( $n$ is an integer). These nuclei are chosen due to the fact that each single-particle level can be occupied by two neutrons and two protons (see sect. 4). Calculations were also performed for ${ }^{16} \mathrm{O}$ and ${ }^{40} \mathrm{Ca}$, and the results were compared with earlier calculations ${ }^{10}$ ), thus providing a check on the numerical computation. A calculation on the $4 p-4$ h state of ${ }^{16} \mathrm{O}$ is also made. This is of current interest, because the $6.06 \mathrm{MeV}^{+}$state of ${ }^{16} \mathrm{O}$ together 
with the excited $2^{+}, 4^{+}$and $6^{+}$states form a rotational band to a good accuracy. Shell-model calculations including all $2 \hbar \omega$ excitations ${ }^{11}$ ) are not able to explain the $0^{+}$state at $6.06 \mathrm{MeV}$. It is suggested ${ }^{12}$ ) that this statc is of a $4 \mathrm{p}-4 \mathrm{~h}$ nature. In all the nuclei both the prolate, oblate and asymmetric solutions are investigated.

In sect. 2, a brief review of the HF theory is given. In sect. 3, the two-body interaction used is discussed. In sect. 4 , the choice of basis states, approximations involved in the truncation of the basis and the iteration procedure are discussed. In sect. 5, the calculation of two-body matrix elements is explained. In sect. 6 , the results of the calculation are presented with discussion. In sect. 7 are the concluding remarks. Appendix 1 describes the separation of the two-body wave function in relative and centre-of-mass coordinates.

\section{The HF theory}

Only a brief review of the HF theory will be given here. A more detailed description of the HF method can be found elsewhere ${ }^{13}$ ).

The Hamiltonian for a many particle system is,

$$
H=\sum_{\alpha \gamma} T_{\alpha \gamma} c_{\alpha}^{\dagger} c_{\gamma}+\frac{1}{4} \sum_{\alpha \beta \gamma \delta} V_{\alpha \beta \gamma \delta} c_{\alpha}^{\dagger} c_{\beta}^{\dagger} c_{\delta} c_{\gamma}
$$

where $T$ is the kinetic energy operator and $V_{\alpha \beta \gamma \delta}$ the antisymmetrized two-body matrix element ${ }^{13}$ ) of the interaction. The $c$ are fermion creation and annihilation operators. The representation $\alpha, \beta, \ldots$ is arbitrary. The HF trial wave function for an $N$-fermion system is a Slater determinant of $N$-particle wave functions,

where

$$
|\mathrm{HF}\rangle=a_{1}^{\dagger} a_{2}^{\dagger} \ldots a_{N}^{\dagger}|0\rangle
$$

$$
a_{i}^{\dagger}=\sum_{\alpha=1}^{p} A_{\alpha i} c_{\alpha}^{\dagger}
$$

In this expansion only $p$ of the complete set of states $\alpha, \beta, \ldots$ are included. This truncation is for practical reasons and will be discussed in more detail in sect. 4 . The $N$ single-particle wave functions ${ }^{\dagger}|i\rangle$ can be chosen to be orthonormal and the set $|i\rangle$ can be completed by choosing wave functions $|i\rangle$ for $i=N+1, N+2 \cdots p$ so as to be orthonormal to the original $N$ functions and to each other.

The HF problem is one of solving for the coefficients $A$ by minimizing $\langle\mathrm{HF}|\mathrm{H}| \mathrm{HF}\rangle$ with respect to $A$. After minimization one obtains a set of pseudo-eigenvalue equations,

where

$$
\sum_{\gamma} W_{\alpha \gamma} A_{\gamma i}=\varepsilon_{i} A_{\alpha i}
$$

$$
\dagger^{\dagger}|\alpha\rangle=c_{\alpha}^{\dagger}|0\rangle \text { and }|i\rangle=a_{i}^{\dagger}|0\rangle .
$$


The HF potential $\Gamma$ is a function of $A$ and is given by

with

$$
\Gamma_{\alpha \gamma}=\sum_{\beta \delta} V_{\alpha \beta \gamma \delta} \rho_{\delta \beta},
$$

$$
\rho_{\delta \beta}=\sum_{i=1}^{N} A_{\beta i} A_{\delta i} .
$$

The one-body density matrix $\rho$ satisfies the conditions

$$
\rho^{2}=\rho, \quad \operatorname{Tr} \rho=N .
$$

The total energy $E_{0}=\langle\mathrm{HF}|H| \mathrm{HF}\rangle$ is given by

$$
E_{0}=\frac{1}{2}\left[\sum_{i=1}^{N} \varepsilon_{i}+\operatorname{Tr}(T \rho)\right]
$$

The expectation value of any one-body operator $K$ can be expressed as

$$
\langle\mathrm{HF}|K| \mathrm{HF}\rangle=\operatorname{Tr}(\rho K) \text {. }
$$

Thus the HF problem can be considered to be one of obtaining $\rho$ which satisfies eq. (5) and is a solution of eq. (2). Since $\Gamma$ [eq. 3] is a function of $\rho$, an iterative method is used to solve for the eigenvalues and eigenfunctions of $W$. An initial $\rho$ that satisfies eq. (5) is chosen, $\Gamma$ is calculated [eq. (3)] and $W$ is diagonalized. With the eigenfunctions of $W$, a modified $\rho$ is obtained from eq. (4) and the procedure is continued until the values of $A_{z i}$ and $\varepsilon_{i}$ do not change with iteration. In each iteration the particles are filled in the lowest single-particle levels of the Hamiltonian $W$.

\section{The two-nucleon interaction}

The two-nucleon interaction used in the present calculation is a non-local separable potential ${ }^{14}$ )

with

$$
V\left(\boldsymbol{r}_{1}, \boldsymbol{r}_{2} ; \boldsymbol{r}_{1}^{\prime}, \boldsymbol{r}_{2}^{\prime}\right)=-\delta\left(\boldsymbol{R}-\boldsymbol{R}^{\prime}\right) F(r) F\left(\boldsymbol{r}^{\prime}\right),
$$

$$
\begin{aligned}
\boldsymbol{R} & =\frac{1}{2}\left(\boldsymbol{r}_{1}+\boldsymbol{r}_{2}\right), \quad \boldsymbol{r}=\boldsymbol{r}_{1}-\boldsymbol{r}_{2}, \\
F(r) & =\left(4 \pi \alpha^{2}\right)^{-1}(\gamma / m \alpha)^{\frac{1}{2}} \exp \left(-\boldsymbol{r}^{2} / 4 \alpha^{2}\right) .
\end{aligned}
$$

This interaction acts only in relative s-states. The range $\alpha$ is chosen to be the same for the singlet and triplet components of the force. The parameters $\alpha$ and $\gamma$ (dimensionless) are chosen by demanding that the interaction give the binding energy/particle of nuclear matter (in the HF approximation) to be $15.7 \mathrm{MeV}$ at the fermi momentum $k_{\mathrm{F}}=1.42 \mathrm{fm}^{-1}$. The second-order correction to the energy in nuclear matter for this interaction ${ }^{10}$ ) is $-0.2 \mathrm{MeV} /$ particle at $k_{\mathrm{F}}=1.42 \mathrm{fm}^{-1}$. Thus this interaction is suitable for HF calculations. Under the assumption that the range $\alpha$ being the same for triplet and singlet components, the binding energy of nuclear matter (or of finite nuclei) is only a function of $\gamma_{\mathrm{s}}+\gamma_{\mathrm{t}}$ and $\alpha$ (where $\mathrm{s}$ and $\mathrm{t}$ refer to singlet and triplet, respectively). The 
values of the parameters are

$$
\begin{gathered}
\alpha_{s}=\alpha_{t}=1.175 \mathrm{fm}, \\
\gamma_{s}+\gamma_{t}=6.167 .
\end{gathered}
$$

The values of $\alpha$ and $\gamma$ that fit the effective ranges and scattering lengths of singlet and triplet states of deuteron are

$$
\begin{array}{ll}
\alpha_{\mathrm{s}}=0.81 \mathrm{fm}, & \alpha_{\mathrm{t}}=0.64 \mathrm{fm}, \\
\gamma_{\mathrm{s}}=2.3, & \gamma_{\mathrm{t}}=3.55,
\end{array}
$$

but these parameters do not fit nuclear matter. Due to the fact that the range of the force is increased to fit nuclear matter the binding energy of nuclei as a function of mass number do not saturate fast enough to give the observed binding energies of light nuclei ${ }^{4}$ ).

\section{The choice of basis states and iteration procedure}

Since we are interested in deformed solutions, in which one expects the singleparticle wave functions $|i\rangle$ to be eigenfunctions of a deformed well (such as the Nilsson potential), the basis states $|\alpha\rangle,|\beta\rangle \cdots$ are chosen to be anistropic harmonic oscillator functions, viz $\left|n_{x} n_{y} n_{z}\right\rangle$. The quantum numbers $n_{x}, n_{y}, n_{z}$ are the number of oscillator quanta in the $x, y$ and $z$ directions. These states are

$$
\begin{aligned}
\left.\left|n_{x} n_{y} n_{z}\right\rangle=\left(b_{x} b_{y} b_{z}\right)^{\frac{1}{2}\left(\frac{1}{2} n_{x}+n_{y}+n_{z}\right.} n_{x} ! n_{y} ! n_{z} !\right)^{\frac{1}{2}} & \times\left(1 / \pi^{\frac{3}{4}}\right) \exp \left(-\frac{1}{2} b_{x} x^{2}-\frac{1}{2} b_{y} y^{2}-\frac{1}{2} b_{z} z^{2}\right) \\
& \times H_{n_{x}}\left(b_{x} x^{2}\right) H_{n_{y}}\left(b_{y} y^{2}\right) H_{n_{z}}\left(b_{z} z^{2}\right),
\end{aligned}
$$

where $b_{i}=m \omega_{i} / \hbar(i=x, y$ or $z)$ and $H_{n}$ are Hermite polynomials. If a complete set is used in the expansion eq. (1) the $b_{i}$ values are arbitrary. But in practical calculations a truncation of the basis is made and the oscillator parameters are also considered as variational parameters. The size of the basis is decided partly by computational limitations and by investigating the effect of increasing the basis. This point is discussed in detail elsewhere ${ }^{10}$ ).

Due to the fact that each single-particle level can be occupied by two neutrons and two protons (spins up and down), there is a four-fold degeneracy of the single particle levels. The number of occupied single-particle energy levels for $A=4 n$ ( $n$ is an integer) is $n$. This is also the reason for considering only nuclei with $A=4 n$. For other nuclei since there is no unique way of filling the levels one should also take into account configuration mixing which is beyond the realm of HF theory.

The single-particle wave functions $|i\rangle$ are assumed to have parity as a good quantum number. Although this restriction is not necessary, it can be shown to be consistent with the HF conditions ${ }^{15}$ ). Moreover this assumption reduces the size of the matrix $W$ and hence simplify the numerical problem. Since parity is a good quantum number 
for $|i\rangle$, the matrices $W$ and $\rho$ do not connect states of different parity and hence the matrix $W$ assumes a block diagonal form as shown in fig. 1a. The two matrices $W_{\text {even }}$ and $W_{\text {odd }}$ can be diagonalized separately. In physical terms, due to the above assumption, pear-shaped nuclei are eliminated. At the present time there is very little evidence for pear-shaped nuclei.

The iteration procedure begins by an initial choice of $\rho$ which satisfies eq. (5) viz,

$$
\begin{gathered}
\rho^{2}=\rho, \\
4 \operatorname{Tr}\left(\rho_{\text {even }}+\rho_{\text {odd }}\right)=A .
\end{gathered}
$$

As an example the initial $\rho$ for ${ }^{20} \mathrm{Ne}$ is shown in fig. $1 \mathrm{~b}$. The final solution depends on the initial choice of $\rho$. For example the choice of $\rho$ shown in fig. $1 \mathrm{~b}$ leads to the

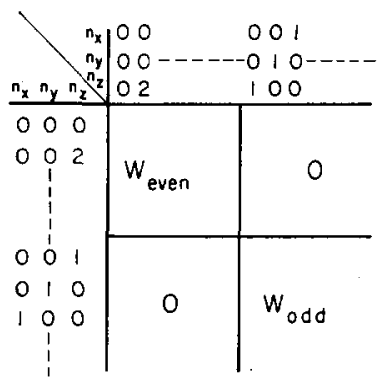

(a)

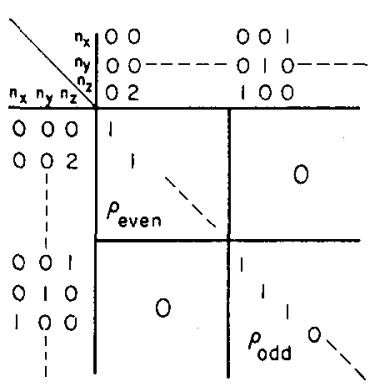

(b)

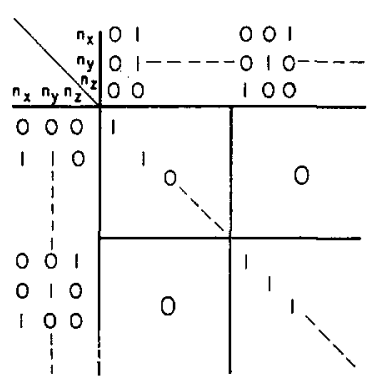

(c)

Fig. 1. The form of the matrices $W$ and $\rho$ under the assumption that parity is a good quantum number for the single-particle wave functions. $b$ and $c$ describe the initial choices of $\rho$ for the prolate and oblate solutions in ${ }^{20} \mathrm{Ne}$.

prolate solution for ${ }^{20} \mathrm{Ne}$, while the $\rho$ shown in fig. 1c gives the oblate solution. The oscillator lengths for the $x$ and $y$ directions are assumed to be the same. For each value of $b_{x} b_{y} b_{z}$, the ratio $b_{x} / b_{z}$ is varied to obtain the minimum energy. For evenparity states, seven basic states are included (all $n_{x}+n_{y}+n_{z}=0$ and 2) and for the odd-parity states 13 states are included (all $n_{x}+n_{y}+n_{z}=1$ and 3). Including more basic states makes the computational part of the problem impractical. But as can be seen from the results the anisotropic oscillator functions are a good approximation to the single-particle wave functions and hence the approximation due to truncation does not introduce appreciable errors in the results.

\section{Two-body matrix element}

Two-body matrix elements of the type $\left\langle n_{x} n_{y} n_{z}, n_{x}^{\prime} n_{y}^{\prime} n_{z}^{\prime}|V| n_{x}^{\prime \prime} n_{y}^{\prime \prime} n_{z}^{\prime \prime}, n_{x}^{\prime \prime \prime} n_{y}^{\prime \prime \prime} n_{z}^{\prime \prime \prime}\right\rangle$ are needed in the calculation of $\Gamma$ [eq. (3)]. The interaction used can be factored in the 
cartesian coordinates,

$$
\begin{array}{r}
V\left(\boldsymbol{r}_{1}, \boldsymbol{r}_{2} ; \boldsymbol{r}_{1}^{\prime}, \boldsymbol{r}_{2}^{\prime}\right)=-\left(4 \pi \alpha^{2}\right)^{-1}(\gamma / m \alpha)^{\frac{1}{2}} \delta\left(X-X^{\prime}\right) \delta\left(Y-Y^{\prime}\right) \delta\left(Z-Z^{\prime}\right) \\
\times F\left(\mathbf{x}, \mathbf{x}^{\prime}\right) F\left(\mathbf{y}, \mathbf{y}^{\prime}\right) F\left(\mathbf{z}, \mathbf{z}^{\prime}\right),
\end{array}
$$

where

$$
\begin{gathered}
X=\frac{1}{2}\left(x_{1}+x_{2}\right), \quad Y=\frac{1}{2}\left(y_{1}+y_{2}\right), \quad Z=\frac{1}{2}\left(z_{1}+z_{2}\right), \quad \mathrm{x}=x_{1}-x_{2}, \\
\quad y=y_{1}-y_{2}, \quad \mathrm{z}=z_{1}-z_{2}, \\
F\left(\mathrm{x}, \mathrm{x}^{\prime}\right)=\exp \left(-\left(\mathrm{x}^{2}+\mathrm{x}^{\prime 2}\right) / 4 \alpha^{2}\right) .
\end{gathered}
$$

Due to this factorization, the two-body matrix elements can be written as a product of three factors of the form

$$
M_{x}=\left\langle n_{x} n_{x}^{\prime}\left|F\left(\mathrm{x}-\mathrm{x}^{\prime}\right) \delta\left(X-X^{\prime}\right)\right| n_{x}^{\prime \prime} n_{x}^{\prime \prime \prime}\right\rangle
$$

and similar expressions for the $y$ and $z$ coordinates. The wave functions $\left|n_{x} n_{x}^{\prime}\right\rangle$ can be expressed in terms of the relative and centre-of-mass coordinates by the following transformation:

$$
\varphi_{n_{x}}\left(b x_{1}^{2}\right) \varphi_{n_{x}^{\prime}}\left(b x_{2}^{2}\right)=\sum_{n_{x}+n_{x}^{\prime}=n+N}\left\langle n_{x} n_{x}^{\prime} \mid n N\right\rangle \varphi_{n}\left(\frac{1}{2} b x^{2}\right) \varphi_{N}\left(2 b X^{2}\right)
$$

where $\varphi$ is the one-dimensional harmonic oscillator wave function ${ }^{16}$ ) and $x$ and $X$ are defined above. The reason for having different oscillator parameters for the centre-of-mass and relative wave function is due to the way $\mathrm{x}$ and $X$ are defined in the present paper. The transformation coefficients $\left\langle n_{x} n_{x}^{\prime} \mid n N\right\rangle$ are given in appendix 1 . The matrix elements [eq. (6)] is given by

where

$$
M_{\times}=\sum_{\substack{n_{x}+n^{\prime} x=n+N \\ n^{\prime \prime} x_{x}+n^{\prime \prime \prime} x=n^{\prime}+N}}\left\langle n_{x} n_{x}^{\prime} \mid n N\right\rangle\left\langle n_{x}^{\prime \prime} n_{x}^{\prime \prime \prime} \mid n^{\prime} N\right\rangle I_{n} I_{n^{\prime}},
$$

$$
\begin{aligned}
I_{n} & =\int_{-\infty}^{+\infty} \varphi_{n}\left(\frac{1}{2} b \mathrm{x}^{2}\right) \exp \left(-\mathrm{x}^{2} / 4 \alpha^{2}\right) \mathrm{dx} \\
& =2^{\frac{3}{4}-\frac{1}{2} n}(\pi b)^{\frac{1}{4}}\left(\alpha /\left(\frac{1}{2} n\right) ! n !\right)\left(b \alpha^{2}-1\right)^{\frac{1}{2} n} /\left(b \alpha^{2}+1\right)^{\frac{1}{2}(n+1)}, \quad n=\text { even } \\
& =0, \quad n=\text { odd. }
\end{aligned}
$$

\section{Results and discussion}

A few general remarks can be made about the single-particle wave functions and energies. In all cases anisotropic oscillator wave functions are a good approximation to the single-particle wave functions (see tables 1-5). Also except for the difference in the oscillator parameters in the $x, y$ and $z$ directions, the structure of the intrinsic wave functions is very similar to the aligned wave functions of the $\mathrm{SU}_{3}$ scheme ${ }^{8}$ ). In the case of axially symmetric solutions $\left({ }^{20} \mathrm{Ne},{ }^{28} \mathrm{Si}\right.$ and $\left.{ }^{36} \mathrm{Ar}\right)$, the single-particle levels can be denoted by the magnetic quantum number $A$. The ordering of the levels 
is qualitatively the same as that of an axially symmetric oscillator with an $l^{2}$ ( $l$ is the orbital angular momentum) term added. But the great depth of the levels show that the single-particle potential is highly non-local. The strength of the $l^{2}$ term changes sign as one goes from ${ }^{16} \mathrm{O}$ to ${ }^{40} \mathrm{Ca}$. This is manifested in the reversal of the position

TABLE 1

Single-particle energies and wave functions for the prolate and oblate solutions of ${ }^{20} \mathrm{Ne},{ }^{28} \mathrm{Si}$ and ${ }^{36} \mathrm{Ar}$

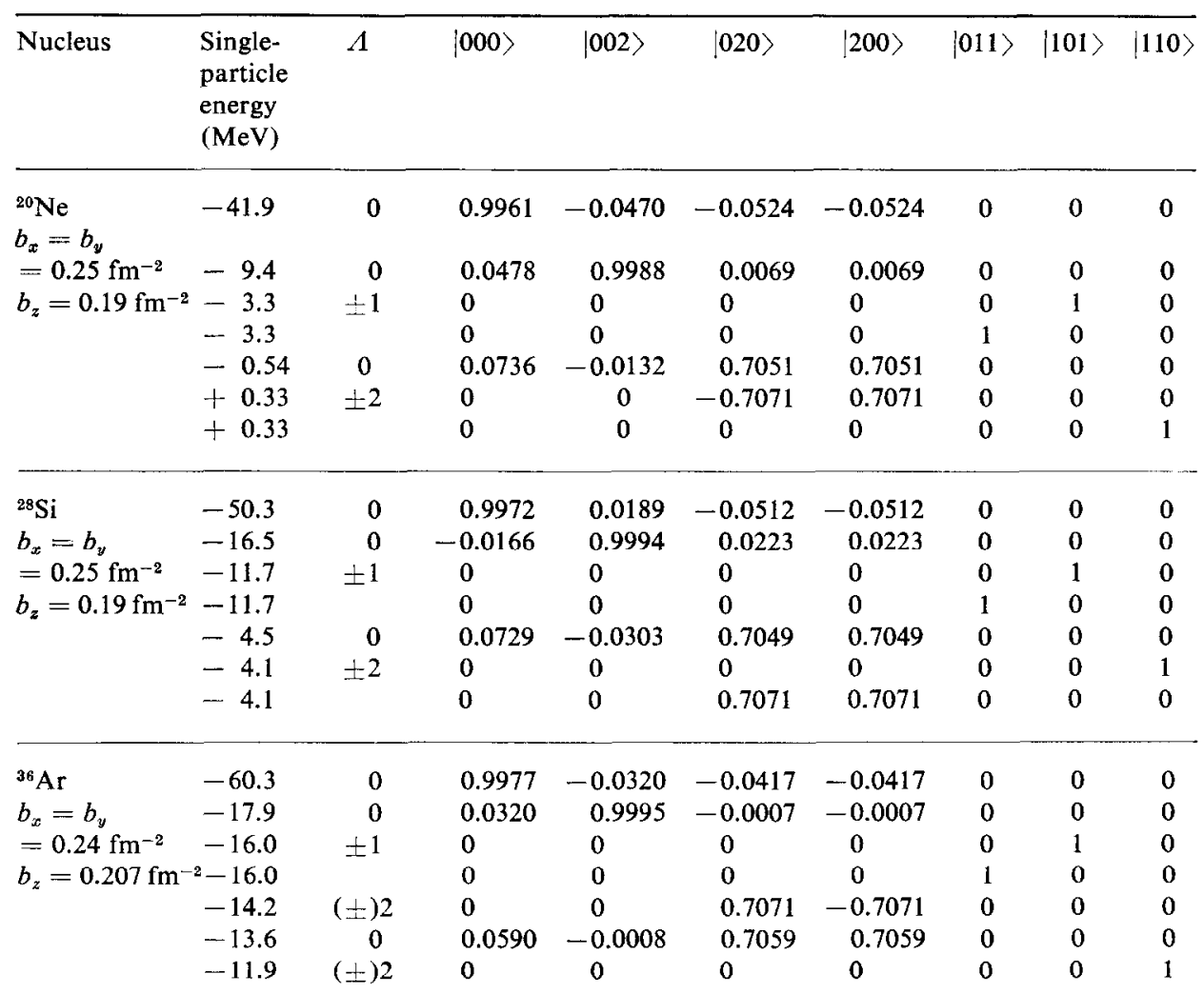

$A$ is the magnetic quantum number and $b=m \omega / \hbar$. In table 1 the even-parity levels of the prolate solution is presented. The even-parity levels for the oblate solutions is given in table 2 . In tables 3 and 4 the odd parity levels for the prolate and oblate solutions are presented respectively.

The particles occupy the lowest levels and each level contains 2 neutrons and 2 protons.

of the $2 \mathrm{~s}$ and $1 \mathrm{~d}$ levels as one goes from ${ }^{16} \mathrm{O}$ to ${ }^{40} \mathrm{Ca}$. This fact is also confirmed by experiments. The qualitative feature of the single-particle levels of ${ }^{28} \mathrm{Si}$ are much more similar to a pure anisotropic oscillator (see fig. 2) than those of ${ }^{20} \mathrm{Ne}$ and ${ }^{36} \mathrm{Ar}$, showing that the $l^{2}$ term is much weaker in the vicinity of ${ }^{28} \mathrm{Si}$. In the case of axially asymmetric solutions $\left({ }^{24} \mathrm{Mg}\right.$ and $\left.{ }^{32} \mathrm{~S}\right)$ the single-particle levels do not have $\boldsymbol{A}$ as a good quantum number, but any one single-particle wave function contains either 
TABLE 2

(see for caption table 1)

\begin{tabular}{|c|c|c|c|c|c|c|c|c|c|}
\hline Nucleus & $\begin{array}{l}\text { Single- } \\
\text { particle } \\
\text { energy } \\
(\mathrm{MeV})\end{array}$ & $A$ & $|000\rangle$ & $|002\rangle$ & $|020\rangle$ & $|200\rangle$ & $|011\rangle$ & $|101\rangle$ & $|110\rangle$ \\
\hline${ }^{20} \mathrm{Ne}$ & -41.3 & 0 & 0.9976 & -0.0397 & -0.0403 & -0.0403 & 0 & 0 & 0 \\
\hline$b_{x}=b_{y}$ & -5.9 & \pm 2 & 0 & 0 & 0 & 0 & 0 & 0 & 1 \\
\hline$=0.216 \mathrm{fm}^{-2}$ & -4.5 & 0 & 0.0591 & 0.0554 & 0.7048 & 0.7048 & 0 & 0 & 0 \\
\hline \multirow{4}{*}{$b_{z}=0.259 \mathrm{fm}^{-2}$} & $2-3.3$ & \pm 2 & 0 & 0 & 0.7071 & -0.7071 & 0 & 0 & 0 \\
\hline & -1.7 & \pm 1 & 0 & 0 & 0 & 0 & 0 & 1 & 0 \\
\hline & -1.7 & & 0 & 0 & 0 & 0 & 1 & 0 & 0 \\
\hline & +0.3 & 0 & 0.0364 & 0.9977 & -0.0407 & $\begin{array}{ll}7 & -0.0407\end{array}$ & 0 & 0 & 0 \\
\hline${ }^{28} \mathrm{Si}$ & -50.9 & 0 & 0.9981 & -0.0449 & -0.0301 & -0.0301 & 0 & 0 & 0 \\
\hline$b_{x}=b_{y}$ & -13.2 & \pm 2 & 0 & 0 & 0 & 0 & 0 & 0 & 1 \\
\hline$=0.206 \mathrm{fm}^{-2}$ & -13.2 & & 0 & 0 & 0.7071 & -0.7071 & 0 & $\mathbf{0}$ & 0 \\
\hline \multirow[t]{4}{*}{$b_{z}=0.29 \mathrm{fm}^{-2}$} & -13.18 & 0 & 0.043 & 0.0104 & 0.7064 & 0.7064 & 0 & $\mathbf{0}$ & 0 \\
\hline & -5.7 & \pm 1 & 0 & 0 & 0 & 0 & 0 & 1 & 0 \\
\hline & -5.7 & & 0 & 0 & 0 & 0 & 1 & 0 & 0 \\
\hline & -0.6 & 0 & 0.0444 & 0.9989 & -0.0087 & -0.0087 & 0 & 0 & 0 \\
\hline${ }^{36} \mathrm{Ar}$ & -60 & 0 & 0.9986 & -0.0425 & -0.0227 & -0.0227 & 0 & 0 & 0 \\
\hline$b_{x}=b_{y}$ & -17.7 & \pm 2 & 0 & 0 & 0 & 0 & 0 & $\mathbf{0}$ & 1 \\
\hline$=0.216 \mathrm{fm}^{-2}$ & -17.7 & & 0 & 0 & 0.7071 & -0.7071 & 0 & 0 & 0 \\
\hline$b_{z}=0.259 \mathrm{fm}^{-2}$ & ${ }^{2}-17.1$ & 0 & 0.0335 & 0.0337 & 0.7063 & 0.7063 & 0 & 0 & 0 \\
\hline & -14.2 & \pm 1 & 0 & 0 & 0 & 0 & 0 & 1 & 0 \\
\hline & -14.2 & & 0 & 0 & 0 & 0 & 1 & 0 & 0 \\
\hline & -8.8 & 0 & 0.0414 & 0.9985 & -0.0248 & -0.0248 & 0 & $\mathbf{0}$ & 0 \\
\hline
\end{tabular}

Table 3

(see for caption table 1)

\begin{tabular}{|c|c|c|c|c|c|c|}
\hline Nucleus & $\begin{array}{l}\text { Single- } \\
\text { particle } \\
\text { energy } \\
(\mathrm{MeV})\end{array}$ & $\boldsymbol{A}$ & Single-particle & wave functions & expanded in the & basis $\left|n_{x} n_{y} n_{z}\right\rangle$ \\
\hline $\begin{array}{l}{ }^{20} \mathrm{Ne} \\
b_{x}=b_{y}\end{array}$ & -23.4 & 0 & $0.9988|001\rangle$ & $-0.0200|003\rangle$ & $-0.0321|021\rangle$ & $-0.0321|201\rangle$ \\
\hline $\begin{array}{l}=0.19 \mathrm{fm}^{-2} \\
b_{z}=0.25 \mathrm{fm}^{-2}\end{array}$ & $\begin{array}{l}-17.0 \\
-17.0\end{array}$ & \pm 1 & $\begin{array}{l}0.9998|010\rangle \\
0.9998|100\rangle\end{array}$ & $\begin{array}{l}-0.0071|030\rangle \\
-0.0071|300\rangle\end{array}$ & $\begin{array}{l}-0.0041|210\rangle \\
-0.0041|120\rangle\end{array}$ & $\begin{array}{l}-0.0192|012\rangle \\
-0.0192|102\rangle\end{array}$ \\
\hline $\begin{array}{l}{ }^{28} \mathrm{Si} \\
b_{x}=b_{y}\end{array}$ & -33.8 & 0 & $0.9987|001\rangle$ & $-0.0279|003\rangle$ & $-0.0294|021\rangle$ & $-0.0294|201\rangle$ \\
\hline $\begin{array}{l}=0.25 \mathrm{fm}^{-2} \\
b_{z}=0.19 \mathrm{fm}^{-2}\end{array}$ & $\begin{array}{l}-23.8 \\
-23.8\end{array}$ & \pm 1 & $\begin{array}{l}0.9977|010\rangle \\
0.9977|100\rangle\end{array}$ & $\begin{array}{l}-0.0346|030\rangle \\
-0.0346|300\rangle\end{array}$ & $\begin{array}{l}-0.0200|210\rangle \\
-0.0200|120\rangle\end{array}$ & $\begin{array}{l}+0.0555|012\rangle \\
+0.0555|102\rangle\end{array}$ \\
\hline $\begin{array}{l}{ }^{36} \mathrm{Ar} \\
b_{x}=b_{y}\end{array}$ & -37.8 & 0 & $0.9975|001\rangle$ & $-0.0591|003\rangle$ & $-0.0274|021\rangle$ & $-0.0274|201\rangle$ \\
\hline $\begin{array}{l}=0.25 \mathrm{fm}^{2} \\
b_{z}=0.207 \mathrm{fm}^{-2}\end{array}$ & $\begin{array}{l}-33.9 \\
-33.9\end{array}$ & \pm 1 & $\begin{array}{l}0.9987|010\rangle \\
0.9987|100\rangle\end{array}$ & $\begin{array}{l}-0.0234|030\rangle \\
-0.0234|300\rangle\end{array}$ & $\begin{array}{l}-0.0407|210\rangle \\
-0.0407|120\rangle\end{array}$ & $\begin{array}{l}-0.0178|012\rangle \\
-0.0179|102\rangle\end{array}$ \\
\hline
\end{tabular}


TABLE 4

(see for caption table 1.)

\begin{tabular}{|c|c|c|c|c|c|c|}
\hline Nucleus & $\begin{array}{l}\text { Single- } \\
\text { particle } \\
\text { energy } \\
(\mathrm{MeV})\end{array}$ & $A$ & Single-particle & wave functions & expanded in the & basis $\left|n_{x} n_{y} n_{z}\right\rangle$ \\
\hline $\begin{array}{l}{ }^{20} \mathrm{Ne} \\
b_{x}=b_{y}\end{array}$ & -20.7 & \pm 1 & $0.9987|100\rangle$ & $-0.0471|300\rangle$ & $+0.0135|120\rangle$ & $-0.01 \quad|102\rangle$ \\
\hline $\begin{array}{c}=0.216 \mathrm{fm}^{-2} \\
b_{z}=0.259 \mathrm{fm}^{-2}\end{array}$ & $\begin{array}{l}-20.7 \\
-16.8\end{array}$ & 0 & $\begin{array}{l}0.9987|010\rangle \\
0.9999|001\rangle\end{array}$ & $\begin{array}{l}-0.0471|030\rangle \\
+0.0148|003\rangle\end{array}$ & $\begin{array}{l}+0.0135|210\rangle \\
-0.0054|021\rangle\end{array}$ & $\begin{array}{l}-0.01|012\rangle \\
-0.0054|201\rangle\end{array}$ \\
\hline $\begin{array}{l}{ }^{28} \mathrm{Si} \\
b_{x}=b_{y}\end{array}$ & -30.3 & \pm 1 & $0.9991|100\rangle$ & $-0.0291|300\rangle$ & $-0.0168|120\rangle$ & $-0.0244|102\rangle$ \\
\hline $\begin{array}{l}=0.206 \mathrm{fm}^{-2} \\
b_{z}=0.29 \mathrm{fm}^{2}\end{array}$ & $\begin{array}{l}-30.3 \\
-20.5\end{array}$ & 0 & $\begin{array}{l}0.9991|010\rangle \\
0.9999|001\rangle\end{array}$ & $\begin{array}{l}-0.0291|030\rangle \\
+0.0153|003\rangle\end{array}$ & $\begin{array}{l}-0.0168|210\rangle \\
-0.0002|021\rangle\end{array}$ & $\begin{array}{l}-0.0244|012\rangle \\
-0.0002|201\rangle\end{array}$ \\
\hline $\begin{array}{l}{ }^{36} \mathrm{Ar} \\
b_{x}=b_{y}\end{array}$ & -37.4 & \pm 1 & $0.9986|100\rangle$ & $-0.0418|300\rangle$ & $-0.0241|120\rangle$ & $-0.0211|102\rangle$ \\
\hline $\begin{array}{l}=0.216 \mathrm{fm}^{-2} \\
b_{z}=0.29 \mathrm{fm}^{-2}\end{array}$ & $\begin{array}{l}-37.4 \\
-31.1\end{array}$ & 0 & $\begin{array}{l}0.9986|010\rangle \\
0.9991|010\rangle\end{array}$ & $\begin{array}{l}-0.0418|030\rangle \\
-0.0427|003\rangle\end{array}$ & $\begin{array}{l}-0.0241|210\rangle \\
+0.0055|021\rangle\end{array}$ & $\begin{array}{l}-0.0211|012\rangle \\
+0.0055|201\rangle\end{array}$ \\
\hline
\end{tabular}

TABLE 5

The single-particle levels and wave functions for ${ }^{24} \mathrm{Mg}$ and ${ }^{32} \mathrm{~S}$ (asymmetric)

\begin{tabular}{|c|c|c|c|c|c|c|}
\hline Nucleus & $\begin{array}{l}\text { Single- } \\
\text { particle } \\
\text { energy } \\
(\mathrm{MeV})\end{array}$ & Parity & Single-particle & wave functions & expanded in the & basis $\left\{n_{x} n_{y} n_{z}\right\rangle$ \\
\hline $\begin{array}{l}{ }^{4} \mathrm{Mg} \\
b_{x}=b_{y} \\
=0.245 \mathrm{fm}^{-2} \\
b_{z}=0.175 \mathrm{fm}^{-2}\end{array}$ & $\begin{array}{r}-45.2 \\
-28.2 \\
-21.5 \\
18.4 \\
-13.0 \\
-9.3 \\
-5.7 \\
-3.6 \\
-1.9 \\
-0.8\end{array}$ & $\begin{array}{l}+ \\
- \\
- \\
+ \\
+ \\
+ \\
+ \\
+ \\
+ \\
+\end{array}$ & $\begin{array}{l}0.9962|000\rangle \\
0.9967|001\rangle \\
0.9991|010\rangle \\
0.9991|100\rangle \\
0.0328|000\rangle \\
|011\rangle \\
|101\rangle \\
0.0406|000\rangle \\
|110\rangle \\
0.0703|000\rangle\end{array}$ & $\begin{array}{r}-0.0315|002\rangle \\
-0.0535|003\rangle \\
-0.0121|030\rangle \\
0.0403|300\rangle \\
+0.9987|002\rangle\end{array}$ & $\begin{array}{l}-0.0749|200\rangle \\
-0.0022|021\rangle \\
-0.0379|210\rangle \\
+0.0110|120\rangle \\
-0.0008|200\rangle \\
+0.1257|200\rangle \\
+0.9892|200\rangle\end{array}$ & $\begin{array}{l}-0.0326|020\rangle \\
-0.0607|201\rangle \\
+0.0133|012\rangle \\
-0.0117|102\rangle \\
+0.0381|020\rangle \\
+0.9905|020\rangle \\
-0.1283|020\rangle\end{array}$ \\
\hline $\begin{array}{l}{ }^{32} \mathrm{~S} \\
b_{x}=b_{y} \\
=0.211 \mathrm{fm}^{-2} \\
b_{z}=0.274 \mathrm{fm}^{-2}\end{array}$ & $\begin{array}{r}-55.5 \\
-35.5 \\
-32.2 \\
-25.8 \\
-16.7 \\
15.5 \\
-14.0 \\
-11.7 \\
-8.2 \\
-4.7\end{array}$ & $\begin{array}{l}+ \\
- \\
- \\
- \\
+ \\
1 \\
+ \\
+ \\
+ \\
+\end{array}$ & $\begin{array}{r}0.9977|000\rangle \\
0.9986|100\rangle \\
0.9983|010\rangle \\
0.9983|001\rangle \\
-0.0019|000\rangle \\
|110\rangle \\
0.0501|000\rangle \\
|101\rangle \\
|011\rangle \\
0.0451|000\rangle\end{array}$ & $\begin{array}{l}-0.0504|020\rangle \\
-0.0236|300\rangle \\
-0.0491|030\rangle \\
-0.0157|003\rangle \\
+0.9980|200\rangle \\
+0.0548|200\rangle\end{array}$ & $\begin{array}{l}+0.0005|200\rangle \\
-0.0443|120\rangle \\
+0.0058|210\rangle \\
-0.0336|021\rangle \\
-0.0546|020\rangle \\
+0.9972|020\rangle \\
+0.0046|020\rangle\end{array}$ & $\begin{array}{l}-0.0448|002\rangle \\
-0.0177|102\rangle \\
-0.0305|012\rangle \\
+0.0440|201\rangle \\
+0.0305|002\rangle \\
-0.0053|002\rangle \\
+0.9985|002\rangle\end{array}$ \\
\hline
\end{tabular}




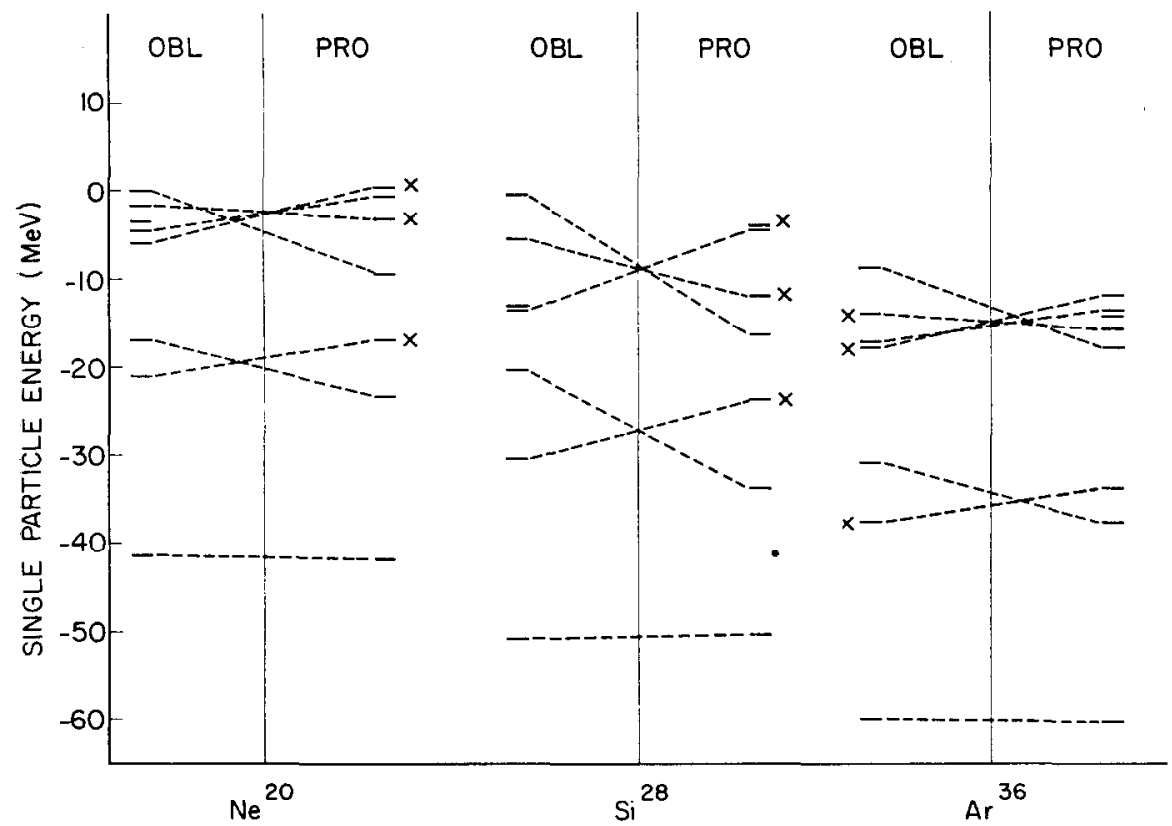

Fig. 2. Single-particle levels for the axially symmetric solutions. Levels of similar structure are connected by dotted lines. The levels marked $x$ are doubly degenerate.

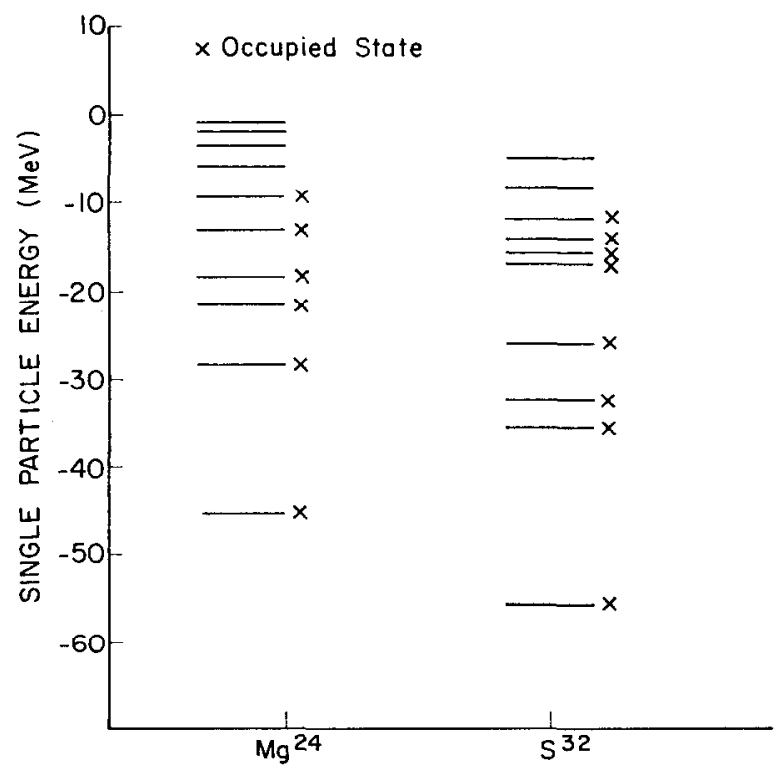

Fig. 3. Single-particle levels for ${ }^{24} \mathrm{Mg}$ and ${ }^{32} \mathrm{~S}$. The solution is asymmetric. 
odd $A$ or even $A$ only. This property is that of the eigenfunctions of an ellipsoidal well. In all nuclei there is an energy gap between the occupied and unoccupied singleparticle levels. This feature is also obtained in some of the earlier calculations ${ }^{17}$ ). This gap is larger for the energetically preferred solution as one would expect. The single-particle levels are shown in figs. 2 and 3. For ${ }^{20} \mathrm{Ne},{ }^{28} \mathrm{Si}$ and ${ }^{36} \mathrm{Ar}$, both the prolate and oblate solutions are given with dotted lines connecting states of similar structure. The level ordering is the same as that of Nilsson ${ }^{1}$ ) (without spin-orbit coupling).

TABLE 6

The single-particle levels and wave functions for the ground state of ${ }^{16} \mathrm{O}$ and ${ }^{40} \mathrm{Ca}$

\begin{tabular}{|c|c|c|c|c|}
\hline Nucleus & $\begin{array}{l}\text { Single- } \\
\text { particle } \\
\text { energy } \\
(\mathrm{MeV})\end{array}$ & $\begin{array}{l}\text { Orbital } \\
\text { angular } \\
\text { momentum } \\
\quad l\end{array}$ & Single-particle & wave functions expanded in the basis $\left|n_{x} n_{y} n_{z}\right\rangle$ \\
\hline $\begin{array}{l}{ }^{16} \mathrm{O} \\
b_{x}=b_{y} \\
b_{z}=0.245 \mathrm{fm}^{-2}\end{array}$ & $\begin{array}{l}-38.3 \\
-15.7 \\
-15.7 \\
-15.7\end{array}$ & 0 & $\begin{array}{l}0.9980|000\rangle \\
0.9999|001\rangle \\
0.9999|010\rangle \\
0.9999|100\rangle\end{array}$ & $\begin{array}{lll}-0.0368[|002\rangle+|020\rangle+|200\rangle] & \\
+0.0099|003\rangle+0.0057|021\rangle & +0.0057|201\rangle \\
+0.0099|030\rangle+0.0057|210\rangle & +0.0057|012\rangle \\
+0.0099|300\rangle+0.0057|120\rangle & +0.0057|102\rangle\end{array}$ \\
\hline $\begin{array}{l}{ }^{40} \mathrm{Ca} \\
b_{x}=b_{y} \\
b_{z}=0.233 \mathrm{fm}^{-2}\end{array}$ & $\begin{array}{r}-65.5 \\
--39.6 \\
-39.6 \\
-39.6 \\
-18.2 \\
18.2 \\
-18.2 \\
-18.2 \\
-18.2 \\
-17.8\end{array}$ & 0 & $\begin{array}{l}0.9986|000\rangle \\
0.9987|100\rangle \\
0.9987|010\rangle \\
0.9987|001\rangle \\
|110\rangle \\
101\rangle \\
011\rangle \\
0.2828|200\rangle \\
0.7660|200\rangle \\
0.0529|000\rangle\end{array}$ & $\begin{array}{l}-0.0306[|002\rangle+|020\rangle+|200\rangle] \\
-0.0394|300\rangle-0.0227[|120\rangle+|102\rangle] \\
-0.0394|030\rangle-0.0227[|210\rangle+|012\rangle] \\
-0.0394|003\rangle-0.0227[|021\rangle+|201\rangle]\end{array}$ \\
\hline
\end{tabular}

The solutions are spherical.

Since we did not separate the centre-of-mass motion from the total Hamiltonian, the ground state energy of the nuclei is obtained by subtracting the kinetic energy of the centre of mass, viz.

$$
\left\langle\mathrm{HF}\left|\frac{1}{2 m A}\left(\sum_{i=1}^{A} p_{i}\right)^{2}\right| \mathrm{HF}\right\rangle
$$

from $\langle\mathrm{HF}|H| \mathrm{HF}\rangle$. In fig. 6 the energy/nucleon is plotted as a function of $A^{-\frac{1}{3}}$. The points lie approximately in a straight line as expected from a two-term mass formula

$$
\text { energy } / A=-a_{\mathrm{v}}+a_{\mathrm{s}} A^{-\frac{1}{3}}
$$

where $a_{\mathrm{v}}$ and $a_{\mathrm{s}}$ are the volume and surface energy coefficients in the Wcizsäcker mass formula ${ }^{18}$ ). The empherical values for $a_{\mathrm{v}}$ is $15 \mathrm{MeV}$ and $a_{\mathrm{s}}$ is $18 \mathrm{MeV}$. The value 
of $a_{\mathrm{s}}$ in our case is approximately $15 \mathrm{MeV}$ and this is due to the fact that the twonucleon interaction is chosen to fit the volume energy of nuclear matter. But the surface term in our case is $24 \mathrm{MeV}$ which is too large. This is duc to the long range of the interaction. However the qualitative behaviour is reproduced well with a simple force as the one used in the present work.

The intrinsic quadrupole moment

$$
Q_{0}=\frac{1}{2}\left\langle\mathrm{HF}\left|\sum_{i=1}^{A}\left(2 z_{i}^{2}-x_{i}^{2}-y_{i}^{2}\right)\right| \mathrm{HF}\right\rangle
$$

TABLE 7

The ground state energy, the quadrupole moment $Q_{0}$ and the asymmetry parameter $Q_{2}$ for ${ }^{16} \mathrm{O},{ }^{20} \mathrm{Ne}$, ${ }^{24} \mathrm{Mg},{ }^{28} \mathrm{Si},{ }^{32} \mathrm{~S},{ }^{36} \mathrm{Ar}$ and ${ }^{40} \mathrm{Ca}$

\begin{tabular}{|c|c|c|c|}
\hline Nucleus & $\begin{array}{l}\text { Ground state } \\
\text { energy }(\mathrm{MeV})\end{array}$ & $\begin{array}{l}Q_{0} \\
\text { (b) }\end{array}$ & $\begin{array}{l}Q_{2} \\
\text { (b) }\end{array}$ \\
\hline${ }^{16} \mathrm{O}$ & -87.0 (spherical) & 0 & 0 \\
\hline${ }^{20} \mathrm{Ne}$ & $\begin{array}{l}-105.4 \text { (prolate) } \\
-95.3 \text { (oblate) }\end{array}$ & $\left.+0.61(0.68)^{a}\right)$ & 0 \\
\hline${ }^{24} \mathrm{Mg}$ & 130.2 (asymmetric) & $\left.+0.77(0.80)^{a}\right)$ & -0.14 \\
\hline${ }^{28} \mathrm{Si}$ & $\begin{array}{l}-165.9 \text { (oblate) } \\
-165.6 \text { (prolate) }\end{array}$ & $\left.-0.83(0.66)^{\mathrm{a}}\right)$ & 0 \\
\hline${ }^{32} \mathrm{~S}$ & -200.3 (asymmetric) & -0.72 & +0.19 \\
\hline${ }^{36} \mathrm{Ar}$ & $\begin{array}{l}-246.5 \text { (oblate) } \\
-240.0 \text { (prolate) }\end{array}$ & -0.57 & 0 \\
\hline${ }^{40} \mathrm{Ca}$ & -300.2 (spherical) & 0 & 0 \\
\hline
\end{tabular}

a) Experimental numbers are from refs. ${ }^{19,26}$ ). The sign of $Q_{0}$ is not determined in these experiments.

and the asymmetry factor

$$
Q_{2}=\left\langle\mathrm{HF}\left|\sum_{i=1}^{A}\left(x_{i}^{2}-y_{i}^{2}\right)\right| \mathrm{HF}\right\rangle
$$

are given in table 7 and fig. 5. There is an abrupt change in the sign of $Q_{0}$ near the middle of the shell and this is probably also supported by experiments ${ }^{19}$ ). The order of magnitude of $Q_{0}$ also agrees exceedingly well with experimental numbers ${ }^{19}$ ). Another interesting feature is the remarkable similarity of these results to those of an earlier calculation ${ }^{5}$ ) in which only nucleons outside the ${ }^{16} \mathrm{O}$ core were considered and the two nucleon interaction used in ref. ${ }^{5}$ ) is very different from ours.

The results for each nucleus with the initial choice of $\rho$ will be discussed below. In giving the initial $\rho$ we shall present the levels that are assumed to be filled. The matrix elements of $\rho$ between filled levels is 1 and all other matrix elements are zero. For example in the case of ground state of ${ }^{16} \mathrm{O}$ initially the states $|000\rangle,|100\rangle,|010\rangle$ and $|001\rangle$ are assumed to be filled. This implies that

$$
\left\langle n_{x} n_{y} n_{z}|\rho| n_{x} n_{y} n_{z}\right\rangle=1,
$$




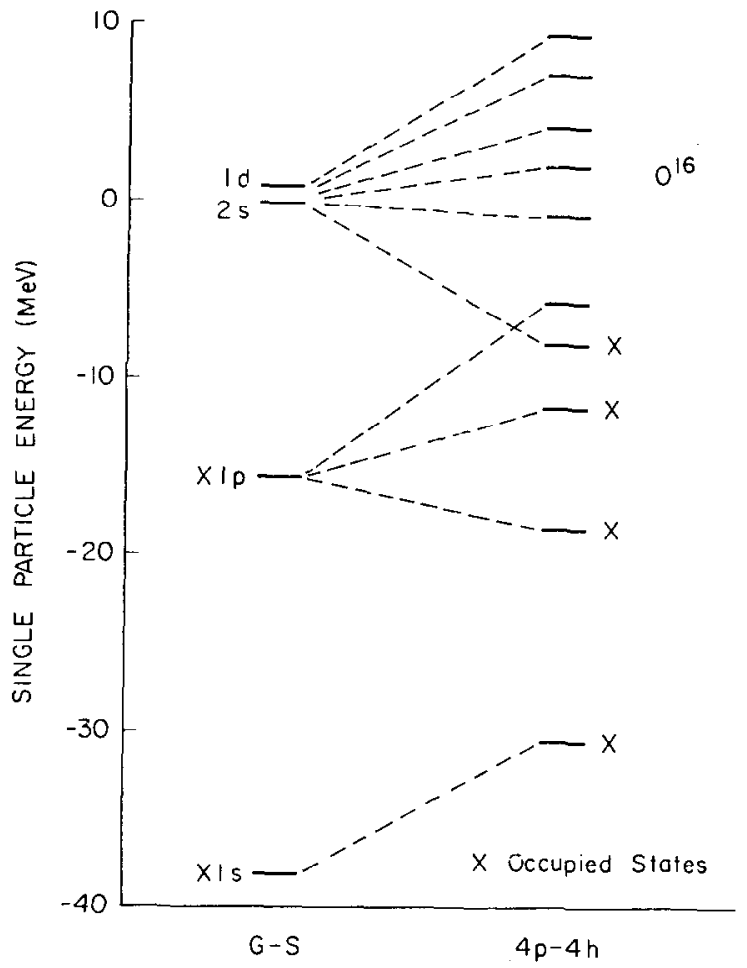

Fig. 4. Single-particle levels for the ground state and the $4 \mathrm{p}-4 \mathrm{~h}$ state of ${ }^{16} \mathrm{O}$. The dotted lines connect states of similar structure.

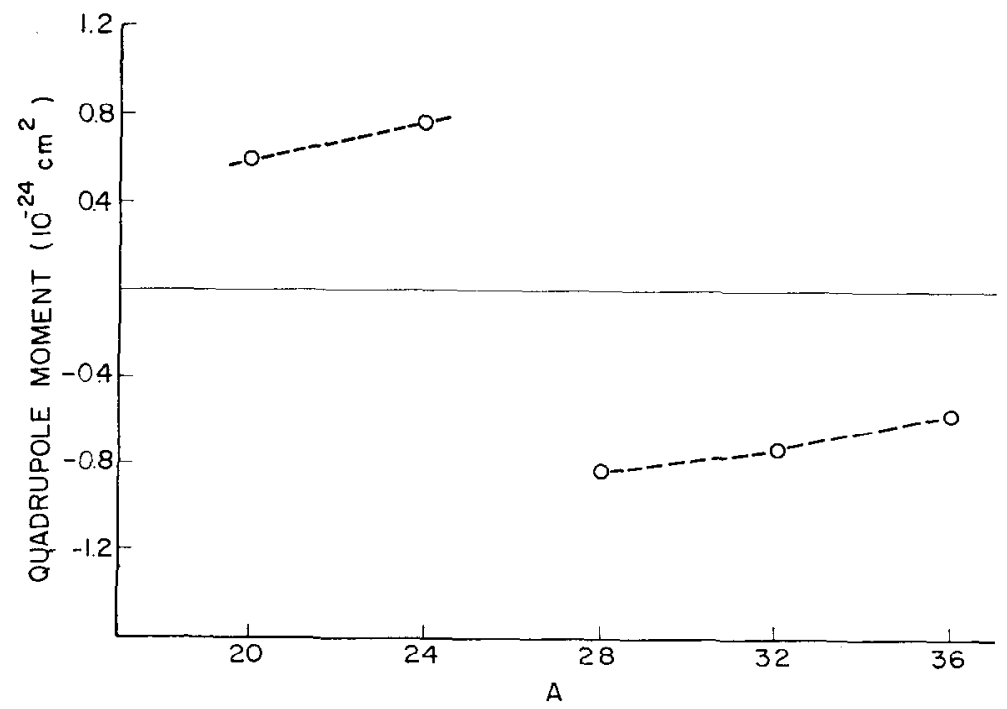

Fig. 5. The intrinsic quadrupole moment $Q_{0}$ versus mass number $A$. 
if $\left|n_{x} n_{y} n_{z}\right\rangle$ is any one of the four levels above. In all nuclei except the $4 \mathrm{p}-4 \mathrm{~h}$ state of ${ }^{16} \mathrm{O}$, the above four levels are filled in the initial choice of $\rho$ and hence only the levels in addition to these will be given.

\subsection{THE ${ }^{16} \mathrm{O}$ AND ${ }^{40} \mathrm{Ca}$ CALCULATION}

In an earlier paper ${ }^{4}$ ) the results of an $\mathrm{HF}$ calculation for ${ }^{16} \mathrm{O}$ and ${ }^{40} \mathrm{Ca}$ were reported. In ref. ${ }^{4}$ ), the nuclei were assumed to be spherical and the single-particle wave functions were expanded in terms of harmonic oscillator functions in spherical

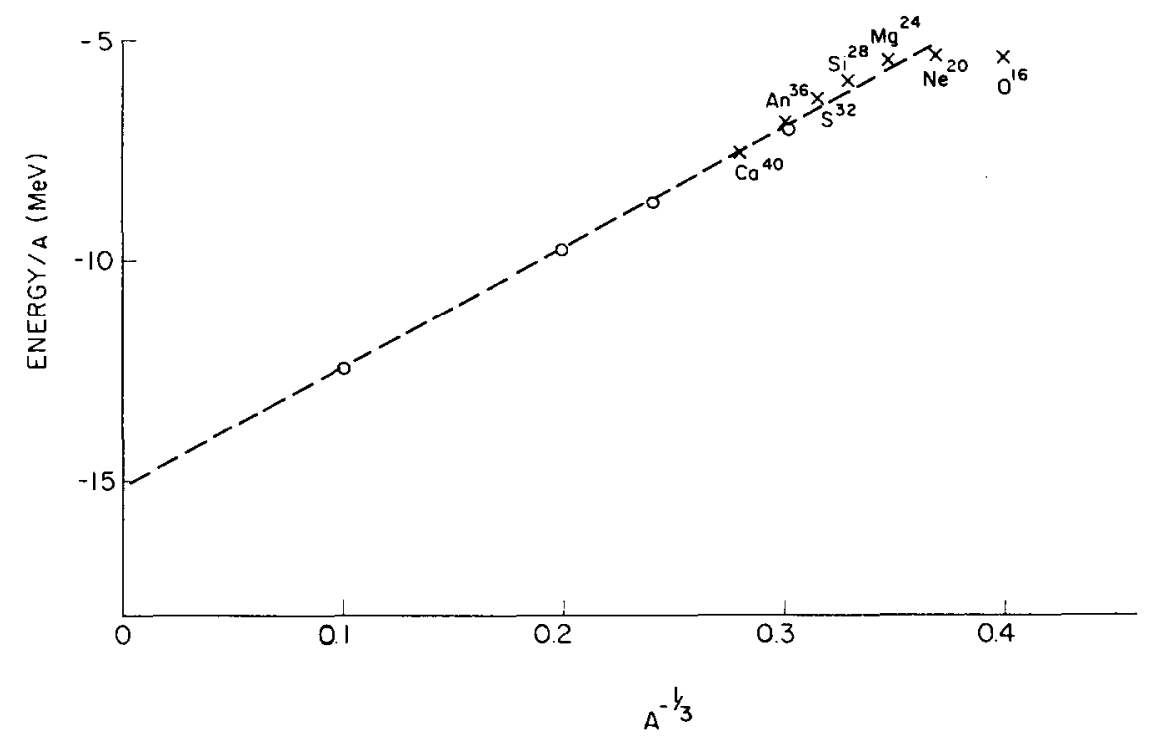

Fig. 6. Energy/nucleon versus $A^{-\frac{1}{3}}$.

coordinates. These results are reproduced in the present calculation (table 6). For ${ }^{40} \mathrm{Ca}$ initially, in addition to the four levels mentioned above, the states $|200\rangle,|020\rangle$, $|002\rangle,|110\rangle,|101\rangle,|011\rangle$ are taken to be filled. Each level is occupied by two neutrons and two protons. The HF single-particle levels (table 6) have both orbital quantum number and magnetic quantum number as good quantum numbers and the levels are $(2 l+1)$ fold degenerate showing that the solutions are spherical.

\subsection{THE ${ }^{20} \mathrm{Ne}$ CALCULATION}

In the case of ${ }^{20} \mathrm{Ne}$ both prolate and oblate solutions are obtained. The initial choices of $\rho$ are shown in figs. $1 \mathrm{~b}$ and $\mathrm{c}$. For the prolate case the state $|002\rangle$ is filled and for the oblate case $|110\rangle$ is filled. The prolate solution is preferred by $10.1 \mathrm{MeV}$.

\subsection{THE ${ }^{24} \mathrm{Mg}$ CALCULATION}

The energetically lowest solution for ${ }^{24} \mathrm{Mg}$ is obtained for the initial choice of $\rho$ in which $|002\rangle$ and $|011\rangle$ are filled. The solution is asymmetric. An axially symmetric 
solution can be found by starting with a $\rho$ in which the states $|200\rangle$ and $|020\rangle$ are filled, but this solution lies much higher in energy.

\subsection{THE ${ }^{28} \mathrm{Si}$ CALCULATION}

In ${ }^{28} \mathrm{Si}$ once again two solutions (prolate and oblate) are found. The initial choice of $\rho$ for the prolate solution assumes that $|002\rangle,|011\rangle$ and $|101\rangle$ are filled and that for the oblate solution assumes that $|200\rangle,|020\rangle$ and $|110\rangle$ are filled. The energy for the two solutions differ by only $0.3 \mathrm{MeV}$, the oblate being lower. In such cases the stability of the minima should be investigated and this cannot be done in any simple way in calculations of the present kind. In calculations of equilibrium nuclear deformations using phenomenological single-particle potentials ${ }^{20}$ ), it was found in similar cases that the solutions were unstable against asymmetric deformation. Recently Litherland et al. ${ }^{21}$ ) have suggested a kind of collective motion in which ${ }^{28} \mathrm{Si}$ nucleus is alternately prolate and oblate without passing through a region of spherical symmetry. This is manifested in a doubling of the rotational bands and there seems to be evidence for such a phenomena.

TABLE 8

The single-particle energies and wave functions for the ${ }^{16} \mathrm{O} 4 \mathrm{p}-4 \mathrm{~h}$ state

\begin{tabular}{lclll}
\hline $\begin{array}{l}\text { Single- } \\
\text { particle } \\
\text { energy } \\
(\mathrm{MeV})\end{array}$ & Parity & Single-particle wave functions expanded in the basis $\left|n_{x} n_{y} n_{z}\right\rangle$ \\
\hline-30.4 & - & & & \\
\hline-18.5 & - & $0.9971|000\rangle-0.031|002\rangle$ & $+0.0553|200\rangle$ & $-0.0414|020\rangle$ \\
-11.7 & - & $0.9983|001\rangle-0.0262|003\rangle$ & $-0.0057|021\rangle$ & $+0.0522|201\rangle$ \\
-8.3 & + & $0.9875|010\rangle+0.1521|030\rangle$ & $+0.017|210\rangle$ & $-0.0363|012\rangle$ \\
-5.8 & - & $0.0335|000\rangle+0.9988|002\rangle$ & $-0.0266|200\rangle$ & $+0.0232|020\rangle$ \\
-0.8 & + & $0.9856|100\rangle+0.0920|300\rangle$ & $+0.1313|120\rangle$ & $+0.0532|102\rangle$ \\
\hline
\end{tabular}

The oscillator parameters are $b_{x}=b_{y}=0.29 \mathrm{fm}^{-2}$ and $b_{z}=0.14 \mathrm{fm}^{-2}$. The energy is $-60 \mathrm{MeV}$.

\subsection{THF ${ }^{32}$ S CALCUI ATION}

The energetically lowest solution in ${ }^{32} \mathrm{~S}$ is obtained for an asymmetric shape and the initial choice of $\rho$ has the states $|200\rangle,|020\rangle,|110\rangle$ and $|101\rangle$ filled.

\subsection{THE ${ }^{36} \mathrm{Ar}$ CALCULATION}

In ${ }^{36} \mathrm{Ar}$ two solutions are obtained corresponding to prolate and oblate deformations and the oblate shape is $6.5 \mathrm{MeV}$ lower than the prolate shape. The initial choices of $\rho$ for the prolate and oblate cases have respectively the states $|002\rangle,|011\rangle$, $|101\rangle,|200\rangle,|020\rangle$ and $|200\rangle,|020\rangle,|110\rangle,|101\rangle,|011\rangle$ filled.

\subsection{THE ${ }^{16} \mathrm{O} 4 \mathrm{p}-4 \mathrm{~h}$ STATE}

As mentioned in sect. 1 , the first excited $0^{+}$state in ${ }^{16} \mathrm{O}$ with $2^{+}, 4^{+}$and $6^{+}$form a rotational band to a very good approximation. Shell-model calculations ${ }^{11}$ ) in- 
cluding all $2 \hbar \omega$ excitations have failed to explain the $0^{+}$state at $6.06 \mathrm{MeV}$. It has been suggested ${ }^{12}$ ) that these states are of a $4 \mathrm{p}-4 \mathrm{~h}$ kind. The initial choice of $\rho$ for this case is

$$
\left\langle n_{x} n_{y} n_{z}|\rho| n_{x} n_{y} n_{z}\right\rangle=1,
$$

if $\left|n_{x} n_{y} n_{z}\right\rangle=|000\rangle,|002\rangle,|001\rangle,|010\rangle$. Four particles are lifted from the state $|100\rangle$ to $|002\rangle$. The results are shown in table 8 and fig. 4 . One significant result is that the lowest single-particle level has gone up by $8 \mathrm{MeV}$ from the ${ }^{16} \mathrm{O}$ ground state solution. In previous calculations ${ }^{22}$ ), these four particles were assumed to be inert . The energy of the $4 \mathrm{p}-4 \mathrm{~h}$ state is $27 \mathrm{MeV}$ above the ground state which is much too high to explain the $6.06 \mathrm{MeV}$ state. This is most probably due to the simplified nature of the interaction used in the present calculation. A repulsion in relative odd states plays an important role in this case ${ }^{23}$ ).

\section{Conclusion}

The main conclusion of the present calculations is that the HF method is well suited for nuclear calculations. Even with a very simple two-nucleon interaction, the salient features of nuclei are well reproduced. With a more sophisticated interaction, one can hope to use the HF method and in the case of deformed nuclei a calculation of the spectra can be made using projection techniques ${ }^{24}$ ) which have been applied recently for light nuclei.

The author would like to thank Dr. T. Engeland for reading the manuscript and for several helpful comments. I also thank Drs. N. Sherman and K. T. Hecht for several helpful comments. Conversations with Dr. M. Baranger of Carnegie Institute of Technology have also contributed very much to the present work.

\section{Appendix 1}

In this section we shall describe the transformation of a product wave function of two particles to relative and centre-of-mass coordinates. The wave functions are onedimensional harmonic oscillator functions. The one-dimensional harmonic oscillator wave function can be written in terms of the raising and lowering operators ${ }^{25}$ ) ( $\eta^{\dagger}$ and $\eta$ ) of oscillator quanta. The two-particle wave function can be written as

where

$$
\left|n_{1} n_{2}\right\rangle=\frac{1}{\sqrt{n_{1} ! n_{2} !}}\left(\eta_{1}^{\dagger}\right)^{n_{1}}\left(\eta_{2}^{\dagger}\right)^{n_{2}}|0\rangle
$$

$$
\begin{aligned}
& \eta_{1}^{\dagger}=(2 m \hbar \omega)^{-\frac{1}{2}}\left(p_{1}+i m \omega q_{1}\right), \\
& \eta_{2}^{\dagger}=(2 m \hbar \omega)^{-\frac{1}{2}}\left(p_{2}+i m \omega q_{2}\right) .
\end{aligned}
$$

The momentum and coordinate are denoted by $p$ and $q$, respectively. In eq. (A.1) 
let us set $\hbar=1$ and $m \omega / \hbar=b$, and we obtain

$$
\begin{aligned}
& \eta_{1}^{\dagger}=\frac{p_{1}}{\sqrt{2 b}}+i \sqrt{\frac{1}{2} b} q_{1}, \\
& \eta_{2}^{\dagger}=\frac{p_{2}}{\sqrt{2 b}}+i \sqrt{\frac{1}{2} b} q_{2} .
\end{aligned}
$$

Let us define the centre-of-mass and relative raising operators to be, respectively,

The operator

$$
\begin{aligned}
& \bar{\eta}^{\dagger}=\left(\eta_{1}^{\dagger}+\eta_{2}^{\dagger}\right) / \sqrt{ } 2, \\
& \eta^{\dagger}=\left(\eta_{1}^{\dagger}-\eta_{2}^{\dagger}\right) / \sqrt{ } 2 .
\end{aligned}
$$

$$
\bar{\eta}^{\dagger}=\frac{p_{1}+p_{2}}{\sqrt{2(2 b)}}+i \sqrt{\frac{1}{2}(2 b)} \frac{1}{2}\left(q_{1}+q_{2}\right) .
$$

Thus $\bar{\eta}^{\dagger}$ creates a quanta with oscillator parameter $2 b$ in the state of momentum $p_{1}+p_{2}$ and coordinate $\frac{1}{2}\left(q_{1}+q_{2}\right)$. The relative coordinate operator

$$
\eta^{\dagger}=\frac{\left(p_{1}-p_{2}\right)}{2 \sqrt{ } b}+\frac{1}{2} i \sqrt{b}\left(q_{1}-q_{2}\right) .
$$

The operator $\eta^{\dagger}$ creates a quanta with oscillator parameter $\frac{1}{2} b$ in the state of momentum $\frac{1}{2}\left(p_{1}-p_{2}\right)$ and coordinate $q_{1}-q_{2}$. Thus if the centre-of-mass and relative coordinates are defined as

$$
\begin{aligned}
& Q_{\mathrm{cm}}=\frac{1}{2}\left(q_{1}+q_{2}\right), \\
& Q_{\mathrm{rel}}=\left(q_{1}-q_{2}\right),
\end{aligned}
$$

the oscillator parameter for the relative and centre-of-mass wave functions are modified as shown above.

Now the wave function in the relative and centre-of-mass coordinates is

$$
|n N\rangle=\frac{1}{\sqrt{n ! N !}}\left(\eta^{\dagger}\right)^{n}\left(\bar{\eta}^{\dagger}\right)^{N}|0\rangle .
$$

Eq. (A.2) can also be written as

$$
|n N\rangle=\sum_{n_{1}+n_{2}=n+N}\left\langle n_{1} n_{2} \mid n N\right\rangle\left|n_{1} n_{2}\right\rangle
$$

The condition $n_{1}+n_{2}=n+N$ is necessary for energy conservation. The wave function eq. (A.2) can also be written as

$$
\begin{aligned}
|n N\rangle & =\frac{1}{\sqrt{n ! N !}} \frac{\left(\eta_{1}^{\dagger}-\eta_{2}^{\dagger}\right)^{n}}{2^{\frac{1}{2} n}} \frac{\left(\eta_{1}^{\dagger}+\eta_{2}^{\dagger}\right)^{N}}{2^{\frac{1}{2} N}}|0\rangle \\
& =\frac{1}{\sqrt{n ! N !}} \sum_{k l}\left[\frac{N ! n !(-1)^{l}}{(N-k) ! k !(n-l) ! l !} \frac{\eta_{1}^{\dagger N+n-k-l} \eta_{2}^{\dagger k+l}}{2^{\frac{1}{2} n} 2^{\frac{1}{2} N}}\right] .
\end{aligned}
$$


Comparing eqs. (A.3) and eq. (A.4), we obtain

$$
\left\langle n_{1} n_{2} \mid n N\right\rangle=\sqrt{\frac{n_{1} ! n_{2} !}{2^{n+N} n ! N !}} \sum_{k} \frac{N ! n !(-1)^{n_{2}-k}}{(N-k) ! k !\left(n-n_{2}+k\right) !\left(n_{2}-k\right) !} .
$$

The summation over $k$ goes over positive values for which the factorials are defined.

\section{References}

1) A. Bohr and B. Mottelson, Mat. Fys. Medd. Dan. Vid. Selsk. 27, No. 16 (1953);

S. G. Nilsson, Mat. Fys. Medd. Dan. Vid. Selsk. 29, No. 16 (1955)

2) D. R. Hartree, Proc. Cambridge Phil. Soc. 24 (1928) 111;

V. Fock, Z. Phys. 61 (1930) 126

3) A. M. Green, Nuclear Physics 33 (1962) 218;

F. Tabakin, Ann. of Phys. 30 (1964) 51

4) R. Muthukrishnan and M. Baranger, Phys. Lett. 18 (1965) 160;

K. T. R. Davis, S. J. Kreiger and M. Baranger, to be published

5) J. Bar-Touv and I. Kelson, Phys. Rev. 138 (1965) B1035

6) D. L. Hill and J. A. Wheeler, Phys. Rev. 89 (1953) 1102

7) R. E. Peirls and J. Yaccoz, Proc. Phys. Soc. A70 (1957) 381

8) J. P. Elliott, Proc. Phys. Soc. A245 (1958) 128, 562

9) S. A. Moskowski, Phys. Rev. 99 (1955) 803

10) R. Muthukrishnan, Ph. D. thesis, Carnegie Institute of Technology (1965)

11) S. S. M. Wong, Phys. Lett. 20 (1966) 188

12) G. E. Brown and A. M. Green, preprint.

13) M. Baranger, Cargese lectures on theoretical physics (W. A. Benjamin, New York, 1963)

14) Y. Yamaghuchi and Y. Yamaghuchi, Phys. Rev. 95 (1954) 1628

15) F. Villars, Proc. Int. School Physics Enrico Fermi (1963)

16) L. Pauling and E. B. Wilson, Introduction to quantum mechanics

17) I. Kelson and C. A. Levinson, Phys. Rev. 134 (1964) B269

18) C. F. Von Weizsäcker, Z. Phys. 96 (1935) 431

19) H. E. Gove, Proc. Int. Conf. nuclear structurc, Kingston, (1960)

20) S. A. Moskowski, Handbuch der Physik, Vol. 39 (1957) p. 503

21) A. E. Litherland, P. J. M. Smulders and T. K. Alexander, preprint

22) W. H. Bassichis and G. Ripka, Phys. Lett. 15 (1965) 320

23) J. Hayward, Nuclear Physics 81 (1966) 193;

R. Muthukrishnan, to be published

24) W. H. Bassichis, B. Giraud and G. Ripka, Phys. Rev. Lett. 15 (1965) 980

25) P. A. M. Dirac, The principles of quantum mechanics, p. 136

26) T. Kh. Lemberg, Proc. Second Conf. on reactions between complex nuclei, Gatlinburg, Tennessee (John Wiley, New York) 